\title{
Co-evolution of Phases and Connection Strengths in a Network of Phase Oscillators
}

\author{
Takaaki Aoki ${ }^{1, *}$ and Toshio Aoyagi ${ }^{1}$ \\ ${ }^{1}$ Graduate School of Informatics, Kyoto University, Kyoto 606-8501, Japan
}

(Received 28 July 2008; published 22 January 2009)

\begin{abstract}
We investigate co-evolving dynamics in a weighted network of phase oscillators in which the phases of the oscillators at the nodes and the weights of the links interact with each other. We find that depending on the type of the dynamics of the weights, the system exhibits three kinds of asymptotic behavior: a twocluster state, a coherent state with a fixed phase relation, and a chaotic state with frustration. Because of its structural stability, it is believed that our model captures the essential characteristics of a class of coevolving and adaptive networks.
\end{abstract}

DOI: 10.1103/PhysRevLett.102.034101

Many real-world complex systems studied in physics, chemistry, biology, and the social sciences can be regarded as dynamical networks of active elements in which the coupling connections and the states of the elements evolve simultaneously. For example, recent neurophysiological experiments indicate that the change in the strength of the synaptic coupling between neurons depends on the relative timing of the pre- and postsynaptic spikes [1]. Such activity-dependent change in synaptic transmission is thought to provide a neuronal basis for realizing higher functions of the brain, such as learning and memory. Similarly, it has been found that in many biological and social networks, the evolution of the network topology, including the coupling weights, is significantly affected by the states of the elements, and vice versa [2]. Such coupled dynamical systems often exhibit interesting types of collective behavior, and their networks display certain characteristic properties [3]. Given the wide variety of the systems in which they appear, it is important to elucidate the essential features of such co-evolving dynamics. The distinctive aspect of co-evolving dynamics is that the coupling weights between the nodes and the states of the active elements at the nodes interact and evolve together. We wish to elucidate the types of behaviors that can emerge in such systems and the functional role played by the evolution of the coupling weights. In this study, considering a simple model as a first step, we attempt to make progress toward this goal.

Among many typical types of behavior exhibited by dynamical systems, limit-cycle oscillation is widely observed in real dissipative systems, and coupled limitcycle systems often generate a rich variety of collective behavior. Furthermore, limit-cycle oscillation is structurally stable, and it can be described by a simple model of phase oscillator that is mathematically tractable $[4,5]$. Therefore, it is reasonable to first consider a limit-cycle oscillator as the dynamical unit at the nodes of the network. It is well known that, using the standard reduction technique, a network of $N$ limit-cycle oscillatory system can be reduced to the coupled phase equation
PACS numbers: 05.45.Xt, 05.65.+b, 89.75.Fb

$$
\frac{d \phi_{i}}{d t}=\omega_{i}+\frac{1}{N} \sum_{j} \Gamma_{i j}\left(\phi_{i}-\phi_{j}\right)
$$

where $\phi_{i}$ denotes the phase of the limit-cycle oscillation at the $i$ th node $(i=1, \ldots N)$, and $\omega_{i}$ is its natural frequency. The coupling function $\Gamma_{i j}(\phi)$ here is assumed to take the simple form $\Gamma_{i j}(\phi)=-k_{i j} \sin (\phi+\alpha)$, where $k_{i j}$ denotes the coupling weight of the connection from the $j$ th to the $i$ th oscillator. Under suitable conditions, the parameter $\alpha$ can be regarded as the phase difference induced by a short delay of the coupling (e.g., a synaptic transmission delay) [6].

Next, we propose a dynamical model for the coupling weights $k_{i j}$. Because it is natural that their dynamics only depend on the relative timing of the oscillators, the following is a reasonable form of such a model

$$
\frac{d k_{i j}}{d t}=\epsilon \Lambda_{i j}\left(\phi_{i}-\phi_{j}\right), \quad\left|k_{i j}\right| \leq 1 .
$$

The time scale of this dynamics, represented by $\epsilon^{-1}$, is much longer than that of the dynamics of the oscillators, i.e., $\epsilon \ll 1$. The condition $\left|k_{i j}\right| \leq 1$ implies that if $k_{i j}$ takes a value outside the interval $[-1,1]$, it is immediately set to the appropriate limiting value ( -1 or 1$)$. This limitation is reasonable, because the coupling weight cannot grow indefinitely. We also numerically confirmed that the same result is obtained when soft limitations are imposed by adding a nonlinear term. The function $\Lambda_{i j}$ determines how the changes in the coupling weights depend on the phase differences among the oscillators. In general, $\Lambda_{i j}(\phi)$ is a $2 \pi$ periodic function. For simplicity, taking only the lowest-order Fourier mode into account, we assume the form $\Lambda_{i j}(\phi)=\Lambda(\phi)=-\sin (\phi+\beta)$. The characteristics of $\Lambda(\phi)$ can be controlled by the parameter $\beta$.

With the above assumptions, the model proposed here is finally given by

$$
\begin{aligned}
& \frac{d \phi_{i}}{d t}=1-\frac{1}{N} \sum_{j} k_{i j} \sin \left(\phi_{i}-\phi_{j}+\alpha\right), \\
& \frac{d k_{i j}}{d t}=-\epsilon \sin \left(\phi_{i}-\phi_{j}+\beta\right), \quad\left|k_{i j}\right| \leq 1,
\end{aligned}
$$


where the natural frequencies are assumed to be identical, and we choose, $\omega_{i}=1$, without loss of generality. This system is characterized by the two parameters $\alpha$ and $\beta$ [7]. In the above simple model, the functions $\Gamma(\phi)$ and $\Lambda(\phi)$ are approximated by only the first Fourier mode. However, we have theoretically and numerically confirmed that even if small higher-order modes are included in these functions, the results given below qualitatively unchanged, and that they are structurally stable.

First, we consider the case of a two-oscillator system. In this case, the dynamics can be written in terms of three variables, $\Delta \phi\left(\equiv \phi_{1}-\phi_{2}\right), k_{12}$ and $k_{21}$, as

$$
\begin{gathered}
\frac{d \Delta \phi}{d t}=-k_{12} \sin (\Delta \phi+\alpha)+k_{21} \sin (-\Delta \phi+\alpha), \\
\frac{d k_{12}}{d t}=-\epsilon \sin (\Delta \phi+\beta), \quad \frac{d k_{21}}{d t}=-\epsilon \sin (-\Delta \phi+\beta) .
\end{gathered}
$$

The condition $\epsilon \ll 1$ implies that the time scale for $\Delta \phi$ is much shorter than that for $k_{i j}$, and hence $\Delta \phi$ quickly relaxes to the equilibrium value, $\Delta \phi^{*}$. (The value $\Delta \phi^{*}$ is given by the condition $\tan \left(\Delta \phi^{*}\right)=-\frac{k_{12}-k_{21}}{k_{12}+k_{21}} \tan \alpha$, obtained from $\frac{d \Delta \phi}{d t}=0$.) We can thus eliminate $\Delta \phi$ in Eq. (4). In addition to the fixed point satisfying $\frac{d k_{12}}{d t}=$ $\frac{d k_{21}}{d t}=0$, there is another type of steady state, in which the weights are given by the limiting values, i.e., $k_{i j}= \pm 1$. Owing to the symmetry of the system, there are two types of such steady states: symmetric, with $\left(k_{12}^{*}, k_{21}^{*}\right)=$ $( \pm 1, \pm 1)$, and asymmetric, with $( \pm 1, \mp 1)$. The condition for the stability of these states is $\operatorname{sgn}\left(-\sin \left(\Delta \phi^{*}+\beta\right)\right)=$ $\operatorname{sgn}\left(k_{12}^{*}\right)$ and $\operatorname{sgn}\left(-\sin \left(-\Delta \phi^{*}+\beta\right)\right)=\operatorname{sgn}\left(k_{21}^{*}\right)$. From the above analysis, we obtain the phase diagram appearing in Fig. 1(a). We find that the two-oscillator system exhibits three types of asymptotic states: symmetric, asymmetric, and chaotic. Furthermore, we also obtained the phase diagram for the case of a large number of phase oscillators appearing in Fig. 2(a). Roughly speaking, we found that the system exhibits three types of asymptotic states corresponding to those in the two-oscillator system. Below, we examine the dynamical properties of each state in detail, focusing mainly on the dependence on $\beta$, because the system not strongly depend on $\alpha$.

We first investigate the region $\beta \in(-\pi, 0)$, in which the symmetric state is stable for the two-oscillator system. In this case, the weights of the two directed links have the same limiting value, i.e., $\left(k_{12}, k_{21}\right)=( \pm 1, \pm 1)$, as shown in Fig. 1. Around the center of this region, we have $\Lambda(\Delta \phi) \sim \cos (\Delta \phi)$ and therefore the coupling weight is increased (decreased) when the phase difference of the two oscillators is small (large). In other words, the evolution of the weights obeys a like-and-like (different-and-different) rule. Note that this situation is qualitatively similar to that of Hebbian learning [8]. Consequently, the system exhibits either in-phase synchronization with positive couplings or

(a)

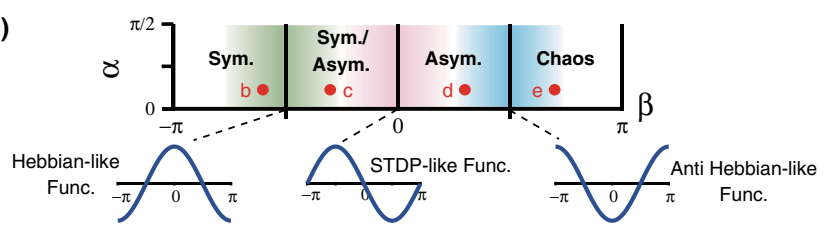

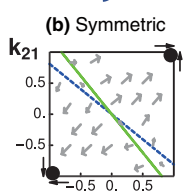

$k_{12}$

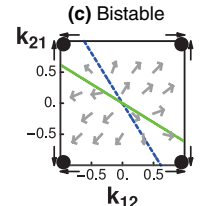

$k_{12}$

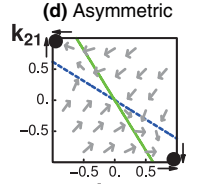

$k_{12}$

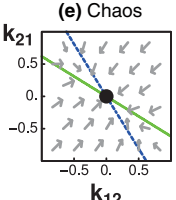

FIG. 1 (color online). (a) Two-parameter phase diagram of a two-oscillator system showing the dependence on $\alpha$ and $\beta$. The asymptotic states can be classified into three types: symmetric, asymmetric and chaotic. In the bistable region, both the symmetric and asymmetric states are stable. The bottom graphs elucidate that the nature of $\Lambda(\Delta \phi)$ changes with the parameter $\beta$. (b)-(e) Phase portraits of the system in the $\left(k_{12}, k_{21}\right)$ plane obtained using an adiabatic approximation for each state. The blue and green lines represent the nullclines for $k_{12}$ and $k_{21}$, respectively. The black circles indicate stable fixed points.

antiphase synchronization with negative couplings, depending on the initial conditions.

For a many-oscillator system in the same parameter region, a two-cluster state appears, as shown in Fig. 2(b). The left graph displays the time development of the order parameters $\left(\left|\frac{1}{N} \sum_{j} e^{i m \phi_{j}}\right|\right.$, with $\left.m=1,2\right)$, and the normalized rate of change of the coupling weights averaged over all $k_{i j}$. The fact that the order parameter for $m=2$ converges to 1 implies that a two-cluster state with antiphase synchronization is realized, and the fact that the rate of change of the total weight converges to zero indicates that the weights become frozen. As a result, a stable two-cluster steady state emerges, and the convergence time depends little on the system size. The middle graph displays the phase distribution after the transient period, in which the oscillators are divided into two clusters. The ratio of the populations of the two clusters generally depends on the initial conditions. From the graph, it is seen that the two clusters with almost equal sizes appear when the initial phases are chosen uniformly from the range $[0,2 \pi)$. From the right graph for the weight matrix $k_{i j}$, we can see that the couplings within a cluster $(\Delta \phi=0)$ are in the state $k_{i j}=$ $k_{j i}=1$, whereas those between different clusters $(\Delta \phi=$ $\pi)$ are in the state $k_{i j}=k_{j i}=-1$. This is essentially the same as the result for the two-oscillator system.

In the region $\beta \in\left(-\frac{\pi}{2}, \frac{\pi}{2}\right)$, asymmetric coupling appears in the two-oscillator system, whereas a bistable region, in which the symmetric coupling is also stable, exists in the region $\beta \in\left(-\frac{\pi}{2}, 0\right)$. Around the center of this region, we have $\Lambda(\Delta \phi) \sim-\sin (\Delta \phi)$, and thus here, the sign of $\Lambda(\Delta \phi)$ is opposite for $\Delta \phi= \pm \Delta \phi^{*}$. This causes changes of the two coupling weights in opposite directions. This situation is essentially the same as that in the case of the rule for spike-timing dependent plasticity 


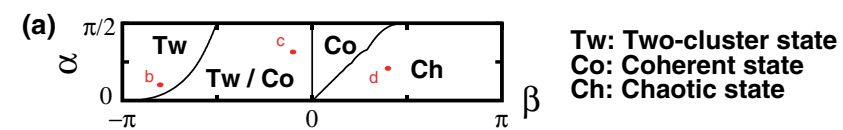

(b) Two-cluster state
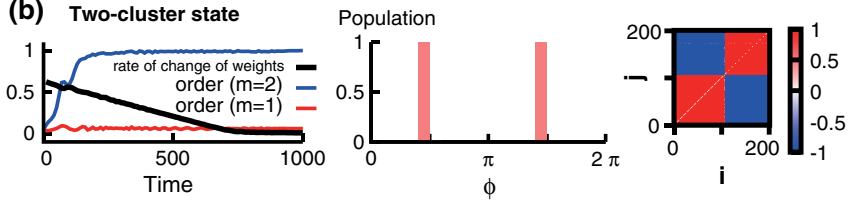

(c) Coherent state
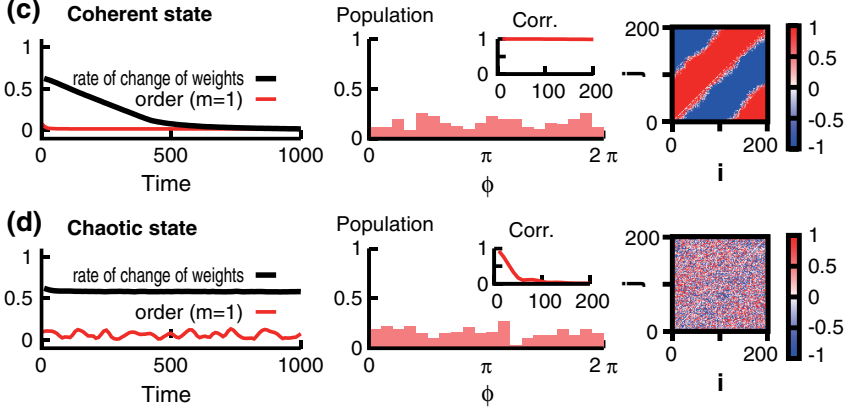

FIG. 2 (color online). (a) Phase diagram of an $N$ oscillator system $(N=200)$. The asymptotic states appearing in the regions depicted are a two-cluster state, a coherent state with a fixed phase relation, and a chaotic state with frustration. (b)(d) Three typical simulation results. (left) Time evolution of the order parameters and the normalized rate of change averaged over all weights. (middle) Distribution of the phase $\phi_{i}$ at $t=$ 1000. The inset displays the autocorrelation function of the phase pattern. (right) The weight matrix $k_{i j}$ in the final state ( $t=$ 1000). The indices $i$ and $j$ of oscillators are arranged in order of increasing phase. The parameter value is $\epsilon=0.005$, and the initial conditions for $k_{i j}$ were chosen randomly from a uniform distribution on $[-1,1]$.

(STDP), reported recently [1]. Because of this property of $\Lambda(\Delta \phi)$, the weights have opposite signs with the same strength, and this results in a $\frac{\pi}{2}$-phase-locking state.

In the case of many oscillators, in contrast to that of two oscillators, it is impossible for any pair of oscillators to synchronize with the phase difference $\Delta \phi^{*}= \pm \frac{\pi}{2}$. As shown in Fig. 2(c), our numerical simulations reveal that the phases of the oscillators are almost uniformly distributed, not organized into clusters. However, the fact that the rate of change of the weights eventually vanishes implies that the system settles into a stable steady state. With respect to the phase patterns, the autocorrelation function $\operatorname{Corr}(\tau)=\left\langle\left|\frac{1}{N} \sum_{j} e^{i \phi_{j}(t)} e^{-i \phi_{j}(t-\tau)}\right|\right\rangle$ does not decay, remaining constant in time. This indicates that the relative phase relations among the oscillators are maintained in a steady state. From these pieces of evidence, we can conclude that the system is in an ordered state, which is referred to as a coherent state with a fixed phase relation. In addition, we found through numerical computations that the relative phases and weights satisfy the relation, $k_{i j}=\operatorname{sgn}\left(-\sin \left(\phi_{i}-\phi_{j}+\beta\right)\right)$. Examining the linear stability of the coherent state with respect to perturbations of the phases, the eigenvalues in the simultaneous limits $N \rightarrow \infty$ and $\epsilon \rightarrow 0$ can be derived as
$\operatorname{Re} \lambda_{k}=-\frac{2}{\pi} \sin (\alpha-\beta) /-\frac{2}{\pi} \sin (\alpha-\beta)-\frac{2}{\pi\left(k^{2}-1\right)} \times$ $(\cos k \beta \sin (\alpha-\beta)+k \sin k \beta \cos (\alpha-\beta))$, when $k$ is even or odd. In Fig. 2(a), we present the stable region of the coherent state determined by this analysis.

For the case of many oscillators, let us examine the region in which both the two-cluster state and the coherent state are unstable. As shown in Fig. 2(d), the system then exhibits a chaotic state with frustration, in which the rate of change of the weights does not converge to 0 and the autocorrelation function for the phase pattern quickly decays to zero. These results imply that both the structure of the weighted network and the phase pattern continue to change with time through their co-evolving dynamics. In fact, in a snapshot of $k_{i j}$, no coherence is observed [right graph in Fig. 2(d)]. This behavior can be understood intuitively as resulting from the appearance of a kind of frustration between the co-evolution of the phases and the coupling weights. In the parameter region, the function $\Lambda(\Delta \phi)$ is qualitatively similar to $-\cos (\Delta \phi)$. This tends to decrease the coupling weights among synchronized oscillators and to increase those among the oscillators of different phases. As a result, both the phase pattern and the network structure are reciprocally destabilized, as illustrated in Fig. 3(a). This evolution rule of the weights has the opposite effect of the like-and-like rule (Hebbian-like) in the case of a two-cluster state. We thus refer to this rule as anti-Hebbian-like rule.
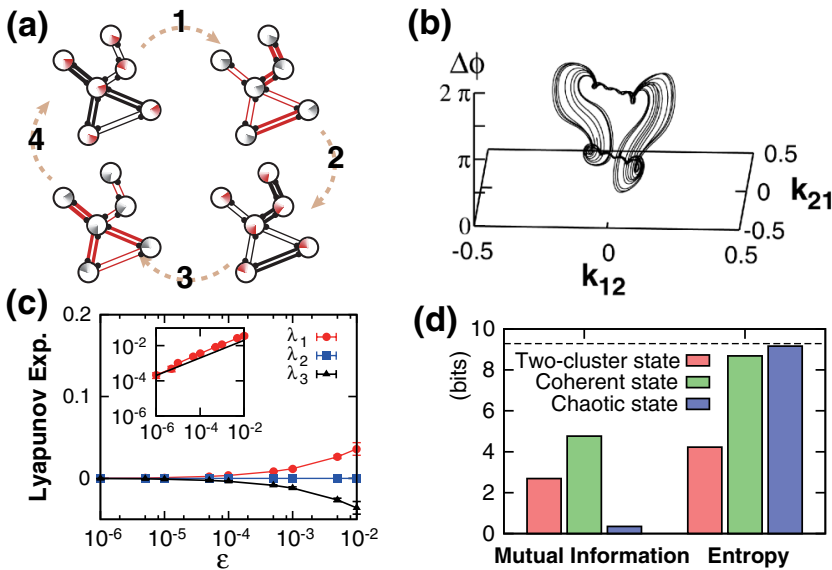

(d)

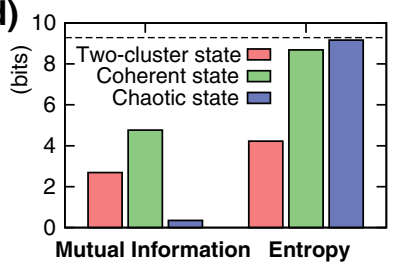

FIG. 3 (color online). (a) A schematic illustration of coevolution of both phase oscillators and network connections. 1. The phase pattern causes the structure of the weighted network to change. 2 . The change undergone by the weights causes a new phase pattern to appear. 3. The change of the phase pattern results in further modulation of the weights of the network. 4. This process repeats. (b) A trajectory for the chaotic state of the two-oscillator system, with $\alpha=0.1 \pi, \beta=0.55 \pi$, and $\epsilon=$ 0.005. (c) Lyapunov exponents as functions of $\epsilon$. The inset displays a log-log plot, with a fitting curve satisfying $\lambda \propto \epsilon^{1 / 2}$. (d) Mutual information between the initial and final phase patterns and the entropy of the final phase pattern for the three asymptotic states. The parameter values here are the same as in (b), (c), and (d) of Fig. 2. The horizontal dashed line represents the maximum attainable entropy for the phase pattern. 
To further investigate this state, let us return to the twooscillator system. For the same parameter region, the phase portrait of the dynamical system reveals that both coupling weights converge to zero [Fig. 1(e)]. In this situation, however, the adiabatic approximation does not hold, even in the limit $\epsilon \rightarrow 0$. As the weights go to zero, the effective time scale for the dynamics of $\Delta \phi^{*}$ becomes larger, and eventually it becomes of the same order as that for the dynamics of $k_{i j}$. Therefore, we must examine the dynamics of the original Eq. (4). Figure 3(b) displays a typical chaotic trajectory for the system given in Eq. (4). As shown in Fig. 3(c), we find that the largest Lyapunov exponent is positive. For a many-oscillator system, we found through numerical computation that the number of positive Lyapunov exponents is proportional to the number of degrees of freedom of the dynamical system, $N^{2}$.

In summary, we have investigated co-evolving dynamics in a weighted network of phase oscillators and found that, depending on the nature of the evolution of the coupling weights, this system can exhibit three distinct types of dynamical behavior: a two-cluster state, a coherent state with a fixed phase relation, and a chaotic state with frustration. A more complete characterization of these states is provided by the mutual information between the initial and final phase patterns and the entropy of final phase pattern [9]. As shown in Fig. 3(d), the mutual information is largest for the coherent state. Because the mutual information is the information that the initial and final states share, the initial phase pattern is most easily inferred from the final one in the case of the coherent state. This suggests that the coherent state can be interpreted as representing a memory of the phase pattern. In context of neural networks, such sequential neural activity embedded in a network organized under STDP learning, has been studied both theoretically and experimentally with regard to the temporal neural coding [10]. For the two-cluster state, the situation is qualitatively similar, except that the entropy is much smaller. This is because the allowed states of an oscillator belonging to the two clusters is restricted to only two possibilities. Therefore, this state is capable of representing a memory of binary data. As stated above, this state is organized under a kind of like-and-like rule. The emergent clustering behavior can be interpreted as a kind of group organization, representing such phenomena as the emergence of communities or opinion formation in social networks, where individuals are organized according to the similarities of their attributes [11]. The related clustering behaviors have been discovered from the viewpoint of the enhancement of synchronization [12]. For the chaotic state, the fact that the mutual information vanishes implies that the information regarding the initial state is lost with time, and the fact that entropy is close to maximal suggests that the system wanders over all or nearly all possible phase patterns. Even though at the present time we cannot give an example of this kind of chaotic state observed in real systems, we believe that such chaotic behavior will be seen in some type of co-evolving systems when compared with our model. Although other important types of systems, such as networks of excitatory units and spatiotemporal systems [13], remain to be studied, we believe that, because of its structural stability, our model will provide a framework for describing essential behavior in co-evolving systems.

This work was supported by CREST, JST, and KAKENHI (20033012， 18047014， 19GS0208) from MEXT and JSPS.

*aoki@acs.i.kyoto-u.ac.jp

[1] G. Q. Bi and M. M. Poo, J. Neurosci. 18, 10464 (1998).

[2] T. Gross and B. Blasius, J. R. Soc. Interface 5, 259 (2008); F. Sorrentino and E. Ott, Phys. Rev. Lett. 100, 114101 (2008); F. Vazquez, V. M. Eguiluz, and M.S. Miguel, Phys. Rev. Lett. 100, 108702 (2008).

[3] S. Boccaletti et al., Phys. Rep. 424, 175 (2006); A.-L. Barabasi and R. Albert, Science 286, 509 (1999); D. J. Watts and S.H. Strogatz, Nature (London) 393, 440 (1998).

[4] Y. Kuramoto, Chemical Oscillations, Waves, and Turbulence (Springer, New York, 1984).

[5] J. A. Acebron el al, Rev. Mod. Phys. 77, 137 (2005).

[6] E. M. Izhikevich, Phys. Rev. E 58, 905 (1998).

[7] Note that this system is invariant under the translation $\left(\alpha, \beta, \phi_{i}\right) \rightarrow\left(-\alpha,-\beta,-\phi_{i}\right)$. Owing to this symmetry, it is sufficient to check the region $\alpha>0$.

[8] D. O. Hebb, The Organization of Behavior (Wiley, New York, 1949).

[9] The initial phases and coupling weights were chosen randomly from $[0,2 \pi]$ and $[-1,1]$, respectively. Then, the chosen phase pattern was hold fixed for a certain period, while the weighs evolved. The asymptotic phase pattern is obtained after a sufficiently large period. We repeated such trials $5.85 \times 10^{6}$ times, and obtained histograms of the phases with bin widths of $2 \pi / N(N=5)$.

[10] T. Aoyagi, Phys. Rev. Lett. 74, 4075 (1995); T. Aoki and T. Aoyagi, Neural Comput. 19, 2720 (2007); E. M. Izhikevich, J.A. Gally, and G. M. Edelman, Cereb. Cortex 14, 933 (2004); K. D. Harris et al., Nature (London) 424, 552 (2003).

[11] G. Palla, A. L. Barabasi, and T. Vicsek, Nature (London) 446, 664 (2007); G. Kossinets and D. J. Watts, Science 311, 88 (2006); J. M. Kumpula et al., Phys. Rev. Lett. 99, 228701 (2007).

[12] J. Karbowski and G. B. Ermentrout, Phys. Rev. E 65, 031902 (2002); P. Seliger, S.C. Young, and L. S. Tsimring, Phys. Rev. E 65, 041906 (2002); D. H. Zanette and A.S. Mikhailov, Europhys. Lett. 65, 465 (2004); Q. Ren and J. Zhao, Phys. Rev. E 76, 016207 (2007); H. Cateau, K. Kitano, and T. Fukai, Phys. Rev. E 77, 051909 (2008); Y. L. Maistrenko et al., Phys. Rev. E 75, 066207 (2007); N. Masuda and H. Kori, J. Comput. Neurosci. 22, 327 (2007).

[13] D. Tanaka, Phys. Rev. Lett. 99, 134103 (2007). 